\title{
Mythos Zentralisierung im Spitalwesen
}

\author{
Grosse Spitäler liefern nicht per se günstigere oder qualitativ bessere Resultate. \\ Im Kanton Bern wurden 13 Kleinspitäler geschlossen. Der finanzielle Erfolg dieser \\ Zentralisierung ist bescheiden.
}

Hans Hoppeler, Jürg Müller, Oliver Richner

Interessenbindung:

Die Autoren sind als Berater für die Firma MKR Partner tätig, die in der Industrie und im öffentlichen Sektor Mandate in Führungsverantwortung, als Projektleiter oder als Berater übernimmt. Im Rahmen dieser Tätigkeit halten sie ein Mandat der «Interessensgemeinschaft Grundversorgung des Kantons Bern», die sich unter anderem für den Erhalt von Regionalspitälern im Kanton Bern einsetzt. Die Darstellung im Artikel repräsentiert aber nur die Meinung der Autoren und ist nicht im Auftrage dieser IG entstanden.

\footnotetext{
* Die Literaturangaben finden sich im Internet unter www. saez.ch $\rightarrow$ Aktuelle Nummer oder $\rightarrow$ Archiv $\rightarrow 2014 \rightarrow 39$.
}

Korrespondenz:

Prof. Dr. med. em. Hans

Hoppeler

Hühnerbühlrain 40

CH-3065 Bolligen

hoppeler[at]ana.unibe.ch

\section{Zentralisierung und Sparpotential im Schweizer Gesundheitswesen}

Die Aufwendungen für das Gesundheitswesen haben 2012 in der Schweiz 68 Milliarden Franken betragen. Eine Untersuchung der Schweizerischen Akademie der Wissenschaften (2012) ortet ein Sparpotential von nicht weniger als $9-11,5 \%$ der Gesamtgesundheitkosten $[1]^{*}$; mögliche Einsparungen, die realisiert werden könnten, ohne dass dabei die Qualität der medizinischen Versorgung leiden würde. Die gewichtigsten Ineffizienzen im schweizerischen Gesundheitswesen sind in der mangelnden Koordination der Leistungserbringung (3 Milliarden), der Überversorgung durch die angebotsinduzierte Nachfrage (1-2 Milliarden) und der Überversorgung durch überhöhte Nachfrage (2 Milliarden) zu orten. Etwas kleiner, aber immer noch beträchtlich $(0,7$ Milliarden) ist das Sparpotential durch Ausnützung von positiven Skaleneffekten bei Schliessung von Kleinspitälern.

Die Zentralisierung des Spitalwesens ist ein politisch gut verankertes Konzept. Basierend auf dem Postulat 09.4239 «Reduktion der Anzahl Spitäler in der Schweiz» und dem Postulat 10.3753 «Klare Kriterien statt Willkür bei Spitallisten» hielt der Bundesrat in seinem Bericht «Grundlagen der Spitalplanung und Ansätze zur Weiterentwicklung» unter anderem fest: «Der Bundesrat seinerseits hat im Rahmen seiner Rechtsprechung in Sachen Spitalplanung neben der Reduktion des Bettenangebotes auch die Reduktion der Anzahl Spitäler unterstützt, dies mit dem Ziel der Erhöhung der Skaleneffizienz (...)» (BAG 2014a [2]).

Zentralisierung im Gesundheitswesen der Schweiz ist allerdings umstritten. Es gibt in der Schweiz 64 Krankenkassen, jede mit einer eigenen Administration, eigener Geschäftsleitung und eigenem Verwaltungsrat. Die Gegner der Einheitskasse fechten mit den Argumenten, eine zentrale Kasse würde die Kosten erhöhen und die Qualität würde wegen fehlender Konkurrenz sinken. Zentralisierung bei Krankenkassen bedeutet demzufolge höhere Kosten und niedrigere Qualität. Im Spitalwesen hingegen fechten die Befürworter der Zentralisierung mit dem Argument, dass dadurch die Kosten gesenkt werden könnten und die Qualität der Dienstleistungen steigen würde. Dieselbe Argumentation für entgegengesetzte Sachverhalte!

\section{Le mythe de la centralisation}

En 2012, les dépenses du système de santé suisse se sont élevées à 68 milliards de francs. L'Académie suisse des sciences évalue le potentiel d'économies à environ $10 \%$ de ce montant, dont une partie serait due à la non-exploitation des économies d'échelle, en d'autres termes, à un nombre trop élevé de petits hôpitaux. La tendance à la fermeture des petits hôpitaux et à la centralisation du secteur hospitalier bénéficie du soutien du milieu politique et elle est généralement bien acceptée auprès des médias et de la population. L'analyse des données suisses montre en effet que les hôpitaux de très petite taille ( $>50$ lits) ne peuvent pas être exploités de manière rentable. D'un autre côté, les coûts augmentent également au sein des hôpitaux de très grande taille, en raison des économies d'échelle négatives et des coûts liés à la complexité. Selon une étude de l'OMS (2014), les économies d'échelle sont déjà pleinement réalisées à partir de 100 à 200 lits. On ne peut cependant pas éviter les coûts liés à la complexité au sein des centres hospitaliers et des hôpitaux universitaires en raison de la diversité des spécialités proposées. En revanche, ces hôpitaux devraient se concentrer sur les cas complexes et difficiles, et laisser les cas sans difficulté particulière aux hôpitaux de premier recours. Centralisation ne signifie pas nécessairement meilleure prise en charge. L'OMS (2014) soutient en effet que les grands hôpitaux ne sont pas meilleurs marché et ne fournissent pas de meilleurs résultats sur le plan qualitatif.

Gibt es objektive Hinweise dafür, dass die Zentralisierung im Spitalwesen auch negative qualitative oder quantitative Effekte haben könnte? Der Kanton Bern liefert Hinweise darauf: Im Kanton Bern 
wurden zwischen 1999 und 2013 nicht weniger als 13 Kleinst- und Kleinspitäler geschlossen. Der finanzielle Erfolg dieser Zentralisierung ist bescheiden, ist doch die Produktivität seiner Spitäler in diesem Zeitraum auf den letzten Platz in der Schweiz abgefallen [3].

\section{Die optimale Spitalgrösse}

Einigkeit in Bezug auf die Beziehung von Spitalgrösse und Produktivität besteht eigentlich nur für sehr kleine Spitäler mit Grundversorgerauftrag $(<50$ Betten). Aufgrund des hohen Fixkostenanteils können diese Kleinstspitäler wohl ökonomisch nicht sinnvoll betrieben werden. Für grössere Spitäler gibt es keine eindeutige Antwort auf die Frage der optimalen Grösse. In einer Untersuchung zur Spitalgrösse berechnen Kristensen et al. 2008 für Dänemark die «optimale Spitalgrösse» auf 275 Betten [4] (mit einer 95\%-Vertrauensgrenze von 130-585 Betten). Sie halten in dieser Studie auch fest, dass der Skaleneffekt bei grösseren Einheiten abnimmt. In einer Folgestudie zeigen Kristensen et al. im Jahr 2010 [5], dass nicht alle Spitalzusammenlegungen Einsparungen bringen und dass man bei zu grossen Einheiten auch mit negativen Skaleneffekten (diseconomy of scale) rechnen muss. Zu einem ähnlichen Schluss kommt Dranove 1998 [6] bei einer Analyse von 14 kalifornischen Spitälern unterschiedlicher Grösse. Er findet, dass eine Zusammenlegung nur für kleine Spitäler sinnvoll ist, dass ab etwa 10000 Fällen pro Jahr keine Einsparungen mehr zu erwarten sind.

Noch klarer hält die WHO 2014 in einer Zusammenfassung des «Health Evidence Networks» fest, dass Einsparungen durch Skaleneffekte im Spitalwesen bei Einheiten zwischen 100 und 200 Betten vollständig realisiert sind [7]. Diese Aussage lässt einen

\section{Abbildung 1}

Aufwand pro Austritt als Funktion der Anzahl Austritte pro Jahr (CMI korrigiert, ohne Investitionskosten, BAG 2014b).

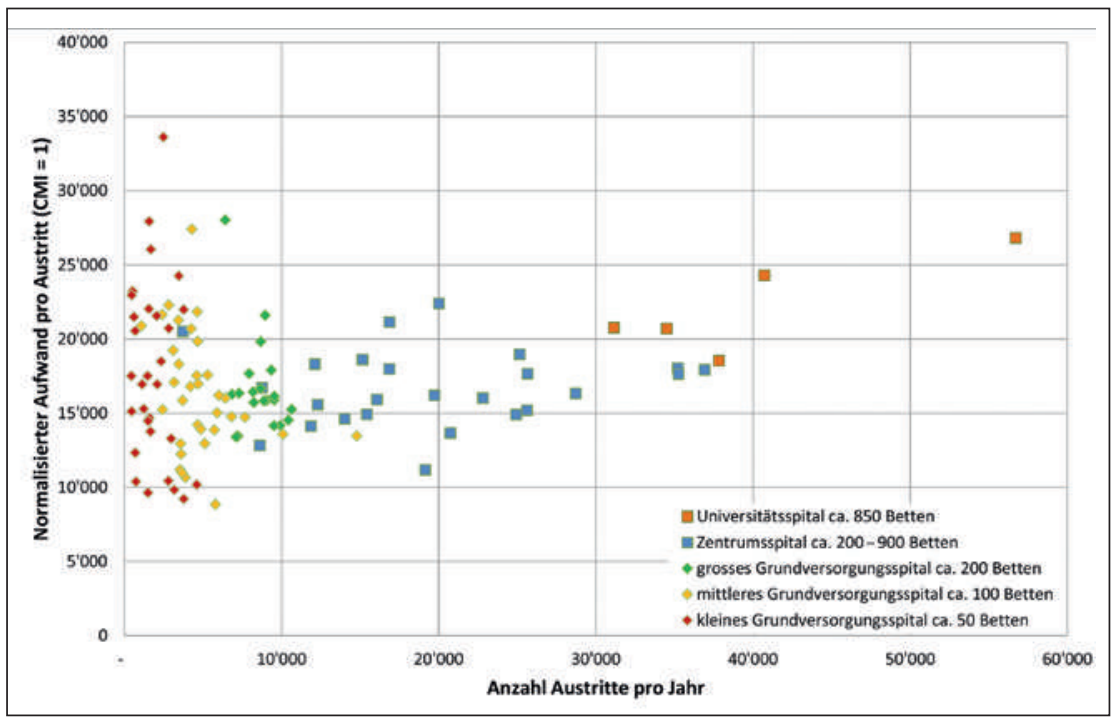

den Sinn einer Zentralisierung grösserer Spitalgebilde hinterfragen.

\section{Skaleneffekte in der Schweiz}

Gerhard Leu hat 2010 einen Bericht zur Effizienz der Schweizer Allgemeinspitäler veröffentlicht [8]. Er stützte sich dabei auf die Statistik des Bundesamts für Gesundheit [9] und definiert die Effizienz eines Spitals als Aufwand an finanziellen Mitteln (ohne Investitionen) pro Austritt (APA). Unter Berücksichtigung von 260 Spitälern fand er, dass nur der CMI (Case-Mix-Index) und die Bettenbelegung die Kosten signifikant beeinflussen, nicht aber die Spitalgrösse. Seine Daten zeigen, dass der Aufwand pro Austritt mit zunehmender Spitalgrösse zunimmt. Korrigiert man den Aufwand pro Austritt mit dem CMI, d. h. berücksichtigt man, dass grössere Spitäler komplexere Fälle behandeln, ergibt sich für alle Spitaltypen (ausser Universitätsspitäler) etwa der gleiche Aufwand pro Austritt. Leu kommt deshalb zum Schluss, dass es bezogen auf den operativen Betriebsaufwand günstiger erscheint, kleine Spitäler zu bauen.

Abbildung 1 zeigt die aktuell verfügbaren Daten in der Schweiz [BAG, 2014; 9]. Wegen der enormen Streuung der Daten wurde auf die Darstellung einer Regressionskurve verzichtet. Es ist aber zu sehen, dass sowohl ganz kleine Spitäler als auch sehr grosse tendenziell höhere Kosten haben. Ein Kostenminimum deutet sich zwischen 8000 und 25000 Fällen an. Man muss also davon ausgehen, dass nicht nur Kleinstspitäler Kostenprobleme haben, sondern dass dies auch für sehr grosse Spitäler zutrifft. Dies ist von Bedeutung, weil das finanzielle «Unheil», das kleine Spitäler anrichten können, relativ harmlos bleibt, bei grossen Spitälern hingegen schon eine geringe Überschreitung des Kostenminimums wegen der hohen Fallzahlen erhebliche Kostenfolgen zeitigt.

\section{Negative Skaleneffekte und Komplexitäts- kosten}

Die höheren Fallkosten sind bei Kleinspitälern im hohen Fixkostenanteil zu suchen, bei den Grossspitälern hingegen in den negativen Skaleneffekten und in Komplexitätskosten. Erstaunlicherweise sind beide Phänomene in der Industrie gut bekannt und belegt, im Gesundheitswesen aber bis jetzt kaum angesprochen.

Negative Skaleneffekte treten dann auf, wenn Organisationen zu gross werden. Als negative Skaleneffekte werden Kosten bezeichnet, die in grossen organisatorischen Einheiten durch zusätzlich benötigte Organisationsebenen anfallen. Diese machen die Kommunikation zwischen den Organisationseinheiten und mit der Führung schwieriger, zeitintensiver und damit teurer. In grossen Einheiten wird im Allgemeinen auch mit einer sinkenden Motivation der Mitarbeiter gerechnet. Es macht deswegen wenig Sinn, Organisationen grösser zu gestalten als not- 
wendig, um Skaleneffekte optimal zu realisieren; diese Grösse wäre für Grundversorgerspitäler (s. [7]) also bei einer Bettenzahl von 100 bis 200 erreicht.

Komplexitätskosten entstehen dann, wenn eine Vielzahl von Leistungen oder «Produkten» innerhalb einer Organisation angeboten werden. Dies ist bei Universitätsspitälern der Fall. Da Universitätsspitäler als letztinstanzliche Versorger fungieren, müssen sie nicht nur alle medizinischen Spezialitäten auf dem Stand des aktuellen Wissens und der neusten Technologie anbieten, sondern auch in der Lage sein, multidisziplinäre Probleme korrekt zu erkennen und zu behandeln. Häufig und kostspieligerweise müssen die Universitätsspitäler auch Fälle peripherer Spitäler übernehme, welche dort zu nicht beherrschbaren (oder allenfalls nicht bezahlbaren) Problemen geführt haben. Es kann davon ausgegangen werden, dass die Komplexitätskosten an den hohen Fallkosten der Universitätsspitäler einen wesentlichen Anteil ausmachen. Es ist offensichtlich, dass besonders die Kostenstruktur der Zentrumsund Universitätsspitäler im aktuellen DRG-System nicht adäquat abgebildet wird [10]. ten, dass Grundversorger- und Privatspitäler vermehrt in die medizinische Aus- und Weiterbildung einbezogen werden müssten. Ein interessantes aktuelles Experiment ist das Zusammenführen eines Universitätsspitals mit einem grossen Grundversorgerspital im Kanton Bern. Es wird sich zeigen, ob (und allenfalls wie) sich negative Skaleneffekte und Komplexitätskosten im grössten Spitalgebilde der Schweiz auswirken.

\section{Spitalgrösse und Qualität der Versorgung}

Bei der Diskussion um die Spitalgrösse wird häufig mit dem Argument der höheren Qualität gefochten. Kleinere Spitäler seien nicht in der Lage, den Qualitätsansprüchen der Medizin zu genügen. Dass «grosses Spital» nicht automatisch «hochwertige Versorgung» bedeutet, hält der bereits zitierte WHO HEN member report [7] fest: «Bigger hospitals are not necessarily better. Research shows that they rarely result in lower costs or better patient outcomes.» Grosse Spitäler sind also nicht eo ipso bessere Spitäler. Wichtiger ist, wie häufig eine Prozedur von einem Arzt oder von einem Team durchgeführt wird. Eine Ana-

\section{«Für grössere Spitäler gibt es keine eindeutige Antwort auf die Frage nach der optimalen Grösse.»}

Interaktiver
Artikel
kollen Sie diesen Artikel
Sie dafür die Kommentar-
funktion in der Online-
Version oder sehen Sie
nach, was Ihre Kolleginnen
und Kollegen bereits
geschrieben haben:
www.saez.ch/
aktuelle-ausgabe/
interaktive-beitraege/

Medizinische Komplexität und die dadurch verursachten Kosten sind zu akzeptieren. Sie sind notwendig, um die relativ kleine Zahl der Patienten, die diese Art von Leistungen benötigen, korrekt zu versorgen. Nicht sinnvoll erscheint, dass Universitätsspitäler auch einen Grossteil der «banaleren» medizinischen Leistungen anbieten. Diese können in den komplexen Strukturen der Universitätsspitäler nicht kostengünstig erbracht werden und sollten den Einheiten, die sich auf die Grundversorgung konzentrieren, vorbehalten bleiben. Konsequenterweise sollte damit auch die Notfallaufnahme eines Universitätsspitals nicht für jedermann frei zugänglich sein, sondern nur für Zuweisungen der Ärzte und der Rettungsequipen offenstehen. Für die spontanen Selbsteinweisungen genügen die Notfallaufnahmen der öffentlichen oder privaten Grundversorgerspitäler. Es bleibe dahingestellt, ob eine solche ökonomisch sinnvolle und medizinisch vertretbare Regelung politisch Akzeptanz finden würde.

Für eine korrekte Leistungsallokation zwischen den Universitäts- und Zentrumsspitälern einerseits und den Grundversorgerspitälern anderseits sind die kantonalen Gesundheitsdirektionen zuständig. Die beschriebene konsequentere Leistungsallokation zwischen Grundversorgung und Spitzenmedizin hätte allerdings Auswirkungen im Bereich der medizinischen Aus- und Weiterbildung. Es würde bedeu- lyse von 2,5 Millionen chirurgischen Prozeduren in den USA in Bezug auf Mortalität und Spitalgrösse zeigt [11], dass Todesfälle bei chirurgischen Interventionen in grossen Einheiten etwas weniger häufig sind. Die Unterschiede sind aber gering, sobald die Zahl der Eingriffe pro Spital 20-30 im Jahr übersteigt. Diese Voraussetzung wird für Herztransplantationen in der Schweiz (30 Transplantationen in 3 Zentren) nicht erfüllt.

Zusammenfassend kann festgehalten werden, dass in der Schweiz eine politisch gestützte Tendenz zur Zentralisierung im Spitalwesen besteht. Soweit es in diesem Zusammenhang um die Schliessung von Kleinstspitälern geht $(<50$ Betten), ist dagegen nichts einzuwenden. Sehr kleine Spitäler kämpfen mit hohen Fixkosten und sind deswegen kaum rentabel zu betreiben. Gestützt auf internationale Daten hält die WHO allerdings fest, dass die Skaleneffekte bei Spitälern mit einer Bettenzahl von 100 bis 200 erschöpft sind. Unerwähnt bleibt, dass grosse Spitäler mit negativen Skaleneffekten und mit Komplexitätskosten zu kämpfen haben. Es ist dringend zu fordern, die bestehende Zentralisierungstendenz kritisch zu hinterfragen und bei Spitalschliessungen nicht nur die Spitalrechnung isoliert zu betrachten, sondern auch Schliessungskosten, Kosten für das Rettungswesen und gemeinwirtschaftliche Leistungen der betroffenen Einheiten auszuweisen [12]. 


\section{Literatur}

1 Schweizerische Akademie der Wissenschaften (2012). Effizienz, Nutzung und Finanzierung des Gesundheitswesens. www.akademien-schweiz.ch/index/Publikationen/Archiv/Berichte.html

2 Bundesamt für Gesundheit (2014a). Grundlagen der Spitalplanung und Ansätze zur Weiterentwicklung. www.bag.admin.ch/dokumentation/publikationen/ 06513/index.html?lang=de

3 Schleiniger R, Blöchliger J. (2012). Menge und Preise der OKP Leistungen. https://www.santesuisse.ch/ datasheets/files/201304031858074.pdf

4 Kristensen T, Olsen KR, Kilsmark J, Pedersen KM. (2008). Economies of scale and optimal size of hospitals: Empirical results for Danish public Hospitals. static.sdu.dk/mediafiles/Files/Om_SDU/ Centre/c_ist_sundoke/Forskningsdokumenter/ publications/Working\%20papers/200813_001.pdf

5 Kristensen T, Bogetoft P, Pedersen, KM. Potential gains from hospital mergers in Denmark. Health Care Management Science. 2010;13:334-5

6 Dranove, D. Economies of scale in non-revenue producing cost centers: implications for hospital mergers. Journal of Health Economics. 1998;17:69-83.
7 WHO, HEN member report (Health Evidence Network) (2014). Are bigger hospitals better? www.euro.who.int/en/data-and-evidence/ evidence-informed-policy-making/publications/ hen-summaries-of-network-members-reports/ are-bigger-hospitals-better

8 Leu G. (2010). Effizienz der Schweizer Allgemeinspitäler. www.gerhardleu.ch/dokumente/ spitaleffizienz-2007.pdf

9 Bundesamt für Gesundheit (2014b). Kennzahlen der Schweizer Spitäler. www.bag.admin.ch/hospital/ index.html?webgrab_path=aHR0cDovL3d3dy5iYWct YW53LmFkbWluLmNoL2t1di9zcGl0YWxzdGF0aXN0aWsvcG9ydGFsX2RILnBocD9sYW5nPWRIJm FtcDtuYXZpZD1renNz\&lang=de

10 Widmer P, Zweifel P. (2014). Gut gemeint ist noch nicht gut getan. NZZ 138, 34. www.nzz.ch/wirtschaft/ gut-gemeint-ist-noch-nicht-gut-getan-1.18324400

11 Birkmeyer JD et al. Hospital volume and surgical mortality in the United States, The New England Journal of Medicine. 2002;346:1128-37.

12 Hoppeler H, Richner O, Müller J. Kosten und Nutzen kleiner Spitäler. Schweiz Ärztezeitung. 2012;93(43):1580-3. 